\title{
Estigma estructural, género e interseccionalidad. Implicaciones en la atención a la salud mental
}

\author{
Jazmín Mora-Ríos, ${ }^{1}$ Natalia Bautista'
}

Artículo original

\section{SUMMARY}

The purpose of this paper is to describe the most common forms and expressions of structural stigma from the perspective of a group of people who attended four treatment centers as a result of having received a psychiatric or neuropsychiatric diagnosis $(n=68)$ and a group of health service providers engaged in these services in Mexico City $(n=95)$. We adopted an approach, based on the intersectionality approach, which involves the interplay between social determinants, including gender, in the processes of social exclusion in mental health. In this qualitative study, the in-depth interview technique was used, for which specific guidelines were designed for providers and users of psychiatric services. The interviews were audio recorded, transcribed and subsequently encoded through a specialized program (Atlas ti, version 7.0). This paper examines the perceptions and experiences of stigma and discrimination related to psychiatric disorders, and other specific aspects related to the care process. Among the findings are the structural nature of discrimination not only directed at people affected by severe mental illness, but also at health personnel. Moreover, the respondents' accounts reveal certain structural barriers that impact the quality of care, particularly for users who have multiple conditions of social vulnerability that go beyond the scope of these service providers, constituting dilemmas for the provider. Lastly, on the basis of the analysis of the various difficulties expressed by the study population in relation to the care services, the main challenges to improving the quality of services in the field of mental health are described.

Key words: Stigma and discrimination, gender and intersectionality, interaction between health care workers and users, health care services, mental health.

\section{RESUMEN}

El objetivo de este trabajo es describir las formas y manifestaciones más comunes del estigma estructural desde la perspectiva de un grupo de personas que acudieron a cuatro centros de atención por un diagnóstico psiquiátrico o neuropsiquiátrico $(\mathrm{n}=68)$, así como de un grupo de trabajadores del área de la salud que laboran en estos servicios en la Ciudad de México (n=95). Adoptamos un enfoque basado en la interseccionalidad que comprende el interjuego que existe entre los determinantes sociales, incluyendo el género, en los procesos de exclusión social en salud mental. En el estudio, de carácter cualitativo, se eligió la técnica de entrevista a profundidad, para lo cual se diseñaron guías específicas en proveedores y usuarios de servicios de atención psiquiátrica. Las entrevistas fueron registradas en audio, posteriormente transcritas y codificadas por medio de un programa especializado (Atlas ti, versión 7.0). Entre los hallazgos destaca el carácter estructural de la discriminación, la cual tiene repercusiones tanto para las personas afectadas por trastornos mentales graves como para el personal de salud. Por otra parte, en las narraciones de los entrevistados se evidencian ciertas barreras estructurales que inciden en la calidad de la atención, particularmente en el caso de aquellos usuarios que presentan múltiples condiciones de vulnerabilidad social que rebasan el alcance de los proveedores de servicios, constituyéndose en dilemas para éstos. Al final se analizan los principales desafíos para mejorar la calidad de la atención en el ámbito de la salud mental.

Palabras clave: Estigma y discriminación, Género e interseccionalidad, interacción personal de salud y usuarios, servicios de atención, salud mental.

\section{INTRODUCCIÓN}

El estigma hacia la enfermedad mental es un fenómeno complejo que se presenta en todas las sociedades y en la última década ha cobrado auge en la investigación, ${ }^{1,2}$ lo cual se explica en gran medida por el incremento que han tenido los padecimientos mentales entre la población de diferentes países y el elevado costo en la atención que generan.
El estigma es un concepto propuesto originalmente por Goffman, quien lo define como una marca o "un atributo profundamente desacreditador"3 que coloca al sujeto en una condición de inferioridad y pérdida de estatus, lo que genera sentimientos de vergüenza, culpabilidad y humillación. El proceso de estigmatización se inicia a través de una serie de señales o marcas, por ejemplo, el color de la piel, la etnia, el género o la condición social, a partir de las cuales

Dirección de Investigaciones Epidemiológicas y Psicosociales. Instituto Nacional de Psiquiatría Ramón de la Fuente Muñíz.

Correspondencia: Jazmín Mora-Ríos. Dirección de Investigaciones Epidemiológicas y Psicosociales. INPRFM. Calzada México-Xochimilco 101, San Lorenzo Huipulco, Tlalpan, 14370, México, D.F. E-mail: morarj@imp.edu.mx 
los sujetos adoptan estereotipos basados en prejuicios que provocan, a su vez, prácticas de discriminación. ${ }^{4}$

Autores como Link y Phelan 5 plantean que gran parte de la producción en esta línea se ha centrado en los aspectos individuales de la experiencia, dejando al margen el análisis de los aspectos estructurales y los determinantes sociales que inciden en los procesos de exclusión. Dichos aspectos conforman el "estigma estructural", también denominado "estigma institucional", que tiene que ver con un conjunto de normas, políticas y procedimientos de entidades públicas o privadas que restringen los derechos y oportunidades de las personas con enfermedades mentales, ${ }^{6}$ legitiman las diferencias de poder y reproducen las inequidades y la exclusión social.

Para estos autores, ${ }^{5}$ la estigmatización involucra cinco procesos psicosociales que se encuentran interrelacionados y que involucran: 1. el etiquetaje, 2. la asignación de estereotipos, 3. la separación, 4. la pérdida de estatus y 5. la discriminación, los cuales ocurren en una situación asimétrica de poder que facilita el que dichos componentes del estigma se desplieguen.

En ese sentido, el estigma estructural constituye un concepto de gran utilidad para el abordaje de los grupos sociales que presentan múltiples condiciones de vulnerabilidad, por ejemplo: personas no heterosexuales, ${ }^{7}$ con $\mathrm{VIH},{ }^{8}$ mujeres recluidas en cárceles, ${ }^{9}$ personas desplazadas por la migración o conflictos bélicos, ${ }^{10}$ así como poblaciones rurales y urbanas que viven en pobreza, ${ }^{11}$ en los cuales intervienen diversos determinantes sociales que se interrelacionan y se expresan en inequidades sociales en el acceso a la salud. Uno de estos determinantes, el género, constituye una variable transversal de carácter estructural que ofrece una explicación más amplia de los modos diferenciales de enfermar en mujeres y hombres, considerando el contexto sociocultural en que se desenvuelven y el sistema social de relaciones establecidas a partir de la interacción entre ambos, sin privilegiar a uno u otro grupo. En ese sentido, el enfoque de interseccionalidad originalmente propuesto por Crenshaw ${ }^{12} \mathrm{y}$ desarrollado por autores como Hankivsky et al., ${ }^{13,14}$ propone que para dar cuenta de la complejidad de los fenómenos sociales, es fundamental el análisis de la interacción simultánea de diversos factores sociales en distintos niveles.

Esta perspectiva se interesa en la inclusión de las políticas públicas en términos de justicia social y reconoce constructos relacionales de la inequidad social, además de que constituye un instrumento teórico de utilidad para examinar de qué manera las relaciones de poder se mantienen y se reproducen. Uno de los principales alcances de este enfoque es que se aleja de cualquier tipo de generalización y propone un nuevo orden de complejidad para entender de qué manera el sexo y el género se interrelacionan con otras dimensiones de inequidad social, en contextos históricos y geográficos específicos, de los cuales los individuos forman parte para crear experiencias únicas en las áreas de salud. ${ }^{13}$ En ese sentido, hablar de interseccionalidad no se reduce a la suma de condiciones de vulnerabilidad sino de la relación dinámica entre los determinantes sociales, el sujeto y su contexto histórico y cultural. ${ }^{13,14}$

El objetivo de este trabajo es describir las formas y manifestaciones más comunes del estigma estructural desde la perspectiva de un grupo de usuarios y proveedores de cuatro centros de tercer nivel de atención. Adoptamos un enfoque basado en la interseccionalidad, que integra el análisis del interjuego que existe entre los determinantes sociales, incluyendo el género como variable transversal, en las experiencias descritas por los participantes en el estudio.

\section{MATERIAL Y MÉTODOS}

La información que se analiza proviene de un estudio transcultural más amplio sobre los procesos de estigmatización en distintos sectores de la población, empleando métodos mixtos. Los aspectos metodológicos se describen en otros trabajos. ${ }^{15-17}$ Se utilizaron formatos de consentimiento informado, garantizando el anonimato y la confidencialidad de la información.

\section{Participantes}

Los datos se basan en una muestra del personal de salud de diferentes disciplinas ( $n=95)$, y de un grupo de usuarios que se encontraban en tratamiento ambulatorio en cuatro centros de atención psiquiátrica ubicados en la Ciudad de México $(n=68)$.

\section{Usuarios de servicios psiquiátricos}

En el cuadro 1 se muestran las características sociodemográficas de los participantes, de los cuales el 73\% eran residentes del Distrito Federal. El rango de edad era de entre 21 y 64 años (SD=11.8), siendo el promedio de 36 años de edad para los hombres $(S D=11.06)$ y de 40 para las mujeres $(S D=11.9)$. El 21\% de los informantes contaba con pareja al momento de la entrevista y la mayor parte compartía vivienda con algún familiar o amigo.

Respecto a las características clínicas, el 90\% tenía un diagnóstico de trastorno mental grave (esquizofrenia, trastorno bipolar, trastorno obsesivo compulsivo y trastorno dual) y en menor proporción, epilepsia (10\%).

El tiempo de duración de sus padecimientos osciló entre menos de un año a cuarenta años, con una media de trece años ( $S D=9.20)$. En promedio tenían nueve años en tratamiento $(\mathrm{SD}=8.24)$ y sólo el $10 \%$ de la muestra tenía menos de un año de recibir atención psiquiátrica. Poco menos de la mitad $(48 \%)$ recibía tratamiento exclusivamente farmacológico, en tanto que el 52\% restante empleaba adicionalmente otros recursos como terapia ocupacional, terapia de pareja, grupal, grupos de apoyo, pláticas de orientación y ejercicios físicos. 
Estigma estructural, interseccionalidad, género y salud mental

Cuadro 1. Características sociodemográficas de los usuarios entrevistados

\begin{tabular}{|c|c|c|c|c|c|c|}
\hline & \multicolumn{2}{|c|}{ Femenino $(n=37)$} & \multicolumn{2}{|c|}{ Masculino $(n=31)$} & \multicolumn{2}{|c|}{ Total } \\
\hline & $\mathrm{F}$ & $\%$ & $\mathrm{~F}$ & $\%$ & $\mathrm{~F}$ & $\%$ \\
\hline \multicolumn{7}{|l|}{ Diagnóstico } \\
\hline - Trastorno mental grave* & 33 & 48.0 & 28 & 42.0 & 61 & 90.0 \\
\hline - Trastorno neuropsiquiátrico (epilepsia) & 4 & 6.0 & 3 & 4.0 & 7 & 10.0 \\
\hline \multicolumn{7}{|l|}{ Edad } \\
\hline - Años & $(21-64)$ & & $(22-63$ & & $(21-64$ & \\
\hline - Media & 40 & & 36 & & 38 & \\
\hline \multicolumn{7}{|l|}{ Estado civil } \\
\hline - Soltera(o) & 28 & 41.0 & 26 & 38.0 & 54 & 79.0 \\
\hline - Con pareja & 9 & 13.0 & 5 & 7.0 & 14 & 21.0 \\
\hline \multicolumn{7}{|l|}{ Escolaridad } \\
\hline - Primaria & 3 & 4.0 & 1 & 1.5 & 4 & 6.0 \\
\hline - Secundaria & 6 & 9.0 & 7 & 10.0 & 13 & 19.0 \\
\hline - Escuela técnica & 14 & 21.0 & 11 & 16.0 & 25 & 37.0 \\
\hline - Licenciatura & 13 & 19.0 & 11 & 16.0 & 24 & 35.0 \\
\hline - Posgrado & 1 & 1.5 & 1 & 1.5 & 2 & 3.0 \\
\hline \multicolumn{7}{|l|}{ Con quién vive } \\
\hline - Solo & 5 & 7.0 & - & - & 5 & 7.0 \\
\hline - Con otras personas & 32 & 47.0 & 31 & 46.0 & 63 & 93.0 \\
\hline \multicolumn{7}{|l|}{ Ocupación } \\
\hline - Empleo & 27 & 40.0 & 22 & 32.0 & 49 & 72.0 \\
\hline - Sin empleo & 10 & 15.0 & 9 & 13.0 & 19 & 28.0 \\
\hline Ingreso mensual & \multicolumn{2}{|c|}{$\begin{array}{c}(100-10,000.00) \\
11(16 \%)\end{array}$} & \multicolumn{2}{|c|}{$\begin{array}{c}(300-12,000.00) \\
17(25 \%)\end{array}$} & \multicolumn{2}{|c|}{$\begin{array}{c}(0-12,000.00) \\
28(41 \%)\end{array}$} \\
\hline
\end{tabular}

*Esquizofrenia, adicciones, trastorno bipolar; Trastorno obsesivo-compulsivo y otros.

\section{Personal de salud}

Participaron informantes provenientes de diferentes disciplinas, principalmente psiquiatras, psicólogos, enfermeros y trabajadores sociales que laboraban en diversos centros de atención en salud mental y adicciones en la Ciudad de México. La mayoría laboraba en instituciones de atención pública (72\%) (cuadro 2). En el cuadro 3 se incluyen algunos datos relacionados con la experiencia laboral de los entrevistados en el área de atención. Con relación al ingreso percibido, es interesante observar que aun cuando es similar el promedio en horas que las mujeres y los hombres destinan a la atención por jornada diaria y semanal, el ingreso tiende a ser más elevado en el caso de los hombres $(\mathrm{T}=0.16, \mathrm{p} \leq .05)$. Este hallazgo tiene implicaciones desde una perspectiva de género, al igual que la tendencia observada en las mujeres para el desempeño laboral en áreas relacionadas con el cuidado de la salud, tales como enfermería y psicología, en contraste con los hombres.

\section{Procedimiento metodológico}

En la investigación se empleó una aproximación basada en métodos mixtos. La recolección de información se llevó a cabo por medio de entrevistas a profundidad con la población, que tuvieron lugar en el periodo comprendido entre enero de 2009 y julio de 2010. El equipo de investigación se conformó por siete entrevistadores provenientes de diversas disciplinas, principalmente del área de psicología, quienes recibieron una capacitación previa en el manejo de las técnicas de recolección de información. Asimismo se llevó a cabo el registro en audio de las entrevistas y posteriormente fueron transcritas para su análisis.

Para efectos de este trabajo se analizaron los siguientes tópicos; en el caso de los usuarios: 1. la percepción del informante respecto al evento que desencadenó su padecimiento, 2. experiencias de estigma y discriminación asociadas con dicho padecimiento, 3. las principales fuentes de estigmatización y 4. sugerencias para reducir el estigma. En el caso de los proveedores de salud, los tópicos analizados fueron: 1. la percepción de los dilemas y dificultades más comunes ligadas con su práctica laboral, 2. percepción de sectores más afectados por los padecimientos de este tipo, 3. experiencias de estigma y discriminación, 4. padecimientos más frecuentemente atendidos en su actividad laboral y 5. sugerencias para reducir el estigma y la discriminación.

\section{Análisis de los resultados}

Se realizó el análisis temático de las entrevistas, mediante un método inductivo. Se utilizó el programa Atlas ti (ver- 
Cuadro 2. Características sociodemográficas del personal de salud entrevistado

\begin{tabular}{|c|c|c|c|c|c|c|}
\hline & \multicolumn{4}{|c|}{ Sexo } & & \\
\hline & \multicolumn{2}{|c|}{$\begin{array}{c}\text { Femenino } \\
(n=61)\end{array}$} & \multicolumn{2}{|c|}{$\begin{array}{c}\text { Masculino } \\
(n=39)\end{array}$} & \multicolumn{2}{|c|}{$\begin{array}{c}\text { Total } \\
(n=95)\end{array}$} \\
\hline & $\mathrm{F}$ & $\%$ & $\mathrm{~F}$ & $\%$ & $\mathrm{~F}$ & $\%$ \\
\hline \multicolumn{7}{|l|}{ Profesión } \\
\hline - Personal no calificado & 6 & 6.3 & - & - & 6 & 6.3 \\
\hline - Medicina general & 2 & 2.1 & 1 & 1.1 & 3 & 3.2 \\
\hline - Trabajo social & 12 & 12.6 & - & - & 12 & 12.6 \\
\hline - Psiquiatría & 13 & 13.7 & 18 & 18.9 & 31 & 32.6 \\
\hline - Enfermería & 15 & 15.8 & 5 & 5.3 & 20 & 21.1 \\
\hline - Psicología & 13 & 13.7 & 10 & 10.5 & 23 & 24.2 \\
\hline \multicolumn{7}{|l|}{ Escolaridad } \\
\hline - Nivel medio superior/técnico & 10 & 10.5 & 1 & 1.1 & 11 & 11.6 \\
\hline - Licenciatura & 17 & 17.9 & 6 & 6.3 & 23 & 24.2 \\
\hline - Posgrado & 34 & 35.8 & 27 & 28.4 & 61 & 64.2 \\
\hline \multicolumn{7}{|l|}{ Área laboral } \\
\hline - Público & 48 & 78.7 & 20 & 58.8 & 68 & 71.6 \\
\hline - Privado & 4 & 6.6 & - & - & 4 & 4.2 \\
\hline - Ambos & 9 & 14.8 & 14 & 41.2 & 23 & 24.2 \\
\hline \multicolumn{7}{|l|}{ Edad } \\
\hline $\begin{array}{l}\text { - Años } \\
\text { - Media }\end{array}$ & \multicolumn{4}{|c|}{$\begin{array}{c}24-68 \\
(39)\end{array}$} & & \\
\hline
\end{tabular}

sión 7.0 ${ }^{18}$ para la organización del análisis cualitativo de la información. Asimismo, se analizó el contenido de las preguntas abiertas.

\section{RESULTADOS}

\section{Percepción en usuarios de servicios psiquiátricos, acerca del evento que desencadenó su padecimiento}

Identificamos seis amplias categorías interrelacionadas cuyos contenidos se muestran en el cuadro 4 . Como se observa, la principal categoría tiene que ver con las condiciones de vulnerabilidad social, que se refiere a la coexistencia de una serie de factores percibidos por el informante, que se interrelacionan y contribuyen al surgimiento del padecimiento. Destacan las precarias condiciones de vida, la violencia y el consumo de sustancias y la falta de una red de apoyo, entre otras. Si bien tanto hombres como mujeres refieren en sus relatos aspectos que no difieren en gran medida respecto a dicha vulnerabilidad social, el género incide en estas experiencias como ocurre, por ejemplo, con la violencia por parte de la pareja, siendo las mujeres víctimas de ésta tanto en el relato de los hombres como en el de las mujeres.

Algo similar ocurre respecto del consumo de alcohol, que sigue siendo una práctica socialmente más aceptada en los hombres que en las mujeres. A continuación se incluye un relato que permite evidenciar las condiciones de vulnerabilidades múltiples y el contexto bajo el cual se refieren:

"...mi ex marido empezó a decirme después de un tiempo de trabajar, que estaban las hijas chicas, que: -ipara qué estudias una carrera?, no sirves para nada y como que esas cosas te van marcando. Si, hay un momento que acabas de decir, no sirvo, no valgo, vas a dar al hospital...(...) te da mucha inseguridad, que es lo que uno pierde con todo esto, el alcohol, el divorcio, las hijas, el no tener trabajo, la muerte de papá...". (Usuaria)

En el siguiente testimonio, que corresponde a un entrevistado, también se observan distintas condiciones de vulnerabilidad social. Específicamente con relación a la violencia, si bien el informante reconoce el impacto de esta experiencia

Cuadro 3. Variables relacionadas con la experiencia laboral del personal de salud, comparación por sexo ( $\mathrm{n}=95)$

\begin{tabular}{|c|c|c|c|c|c|c|c|}
\hline \multirow[b]{2}{*}{ Variables } & \multicolumn{3}{|c|}{ Mujeres } & \multicolumn{3}{|c|}{ Hombres } & \multirow[b]{2}{*}{$\mathrm{p}(\mathrm{t})$} \\
\hline & Media & $\mathrm{DE}$ & Rango & Media & $\mathrm{DE}$ & Rango & \\
\hline Experiencia en salud mental (años) & 11.65 & 8.37 & $0-30$ & 13.60 & 9.02 & $2-39$ & ns \\
\hline Antigüedad en empleo actual (años) & 9.63 & 8.02 & $0-29$ & 10.59 & 8.53 & $0-28$ & ns \\
\hline Atención diaria al usuario (horas) & 5.76 & 2.74 & $0-11$ & 6.85 & 3.38 & $1-20$ & ns \\
\hline Atención semanal al usuario (horas) & 29.45 & 13.81 & $0-56$ & 34.85 & 13.44 & $5-66$ & ns \\
\hline Ingreso mensual & $14,526.67$ & 9491.7 & $1,000-50,000$ & $19,382.35$ & 8861.6 & $8,000-40,000$ & 0.01 * \\
\hline
\end{tabular}

* $0=$ Menos de un año. $p<.05$. 
en su padecimiento, ya que su madre era violentada por su padre, él no se asume como receptor directo de dicha violencia, como en el caso anteriormente descrito:

"Sí, pues cuando era pequeño mi papá tomaba mucho ¿no? y yo me privaba digamos, lloraba por la ausencia de mi mamá, me ponía hasta morado, entonces dicen que una vez convulsioné y eso me pudo haber afectado, el no respirar tanto tiempo y luego este pues yo me acuerdo que mi papá le pegaba a mi mamá(...)luego, cuando yo cumplí 10 años mi papá desarrolla una psicosis maniaco depresiva y entonces empecé a trabajar en el mercado y pues aprendí malos hábitos ¿no?, ...o sea, como ver pornografía y eso. Ya empecé a tener problemas en la escuela y luego a los 16 años...cuando mi abuelito falleció pues empezaron los problemas porque consumí hongos y marihuana y ya según de ahí me enfermé y empecé yo a tener los delirios pero resulta que nunca me curé de eso...". (Usuario)

Identificamos una categoría a la que denominamos "características del individuo" y se refiere a los atributos que desde la percepción del informante contribuyeron al padecimiento, por ejemplo, percibirse de un modo distinto a los demás desde la infancia, tener un comportamiento extraño y aislarse de otras personas, además de la adopción de hábi- tos que consideraron inadecuados: alimentación, sueño, lectura, etc. Si bien los factores genéticos fueron identificados como un detonador de la enfermedad, cabe destacar que los aspectos culturales con relación a las creencias mágico-religiosas, las prácticas esotéricas, así como las decepciones amorosas, también lo fueron.

Por otra parte, la categoría que nombramos como "problemas" incluyó una serie de dificultades laborales, de pareja, académicas, familiares y personales que desde la percepción del entrevistado propiciaron el surgimiento de la crisis. Incorporamos otra categoría bajo la cual se agruparon eventos específicos que rebasan la acción directa del sujeto: asaltos, accidentes y golpes en la cabeza, así como la pérdida de seres queridos.

\section{Experiencias de estigma y discriminación referidas por los usuarios}

El 91\% de los entrevistados reconocieron el estigma y la discriminación que existen en la sociedad hacia las personas

Cuadro 4. Percepción de evento que desencadenó el padecimiento psiquiátrico, según usuarios

\begin{tabular}{|c|c|c|}
\hline & Mujeres & Hombres \\
\hline $\begin{array}{l}\text { Condiciones } \\
\text { de vulnerabilidad } \\
\text { social } \\
\text { (35 menciones) }\end{array}$ & $\begin{array}{l}\text { - Violencia de pareja, consumo de alcohol y drogas, por } \\
\text { subir de peso y problemas emocionales en su familia } \\
\text { - Abuso sexual, no contar con el apoyo de su familia y } \\
\text { falta de recursos económicos } \\
\text { - Pérdidas (muerte, separaciones de padres y de pareja) } \\
\text { - Comportamiento extraño en la infancia y empeora } \\
\text { con la separación de sus padres } \\
\text { - "Vivencias traumáticas en la infancia" } \\
\text { - Antecedentes de enfermedades físicas y mentales en } \\
\text { - Ma familia } \\
\text { - Maltrato por parte de su madre y epilepsia en su } \\
\text { - Proviene de una familia donde existe mucha depre- } \\
\text { sión, violencia de pareja }\end{array}$ & $\begin{array}{l}\text { - Consumo de alcohol y drogas, creció en un ambiente de } \\
\text { consumo, conflictos y saber que depende de sus padres } \\
\text { - Experiencias de negligencia y maltrato en la infancia, } \\
\text { conflicto con sus familiares, violencia del padre hacia su } \\
\text { - madre } \\
\text { - Consumo de substancias en familiares cercanos } \\
\text { - Otros padecimientos crónicos en la familia } \\
\text { - Experiencias negativas en la atención ("le aplicaron mal } \\
\text { - Pra vacuna") } \\
\text { - Experión de sus padres y problemas de pareja }\end{array}$ \\
\hline $\begin{array}{l}\text { Características } \\
\text { del individuo } \\
\text { (18 menciones) }\end{array}$ & $\begin{array}{l}\text { - Por su personalidad solitaria } \\
\text { - Comportamiento extraño en la infancia } \\
\text { - Consumo de alcohol y drogas } \\
\text { - "No comer bien" }\end{array}$ & $\begin{array}{l}\text { - Su comportamiento siempre fue extraño } \\
\text { - Consumo de alcohol y/drogas } \\
\text { - Por su forma de ser } \\
\text { - "Deseo de pertenecer a un grupo de amigos" } \\
\text { - Problemas psicológicos } \\
\text { - Mala alimentación } \\
\text { - Falta de ejercicio } \\
\text { - Falta de lectura }\end{array}$ \\
\hline $\begin{array}{l}\text { Genética } \\
\text { (17 menciones) }\end{array}$ & $\begin{array}{l}\text { - Antecedentes de padecimientos psiquiátricos } \\
\text { - Alteración bioquímica }\end{array}$ & $\begin{array}{l}\text { - Antecedentes de padecimientos psiquiátricos en la familia } \\
\text { - Por alguna razón psicológica que no alcanza a entender } \\
\text { - Por probabilidad estadística, "le tocó" }\end{array}$ \\
\hline $\begin{array}{l}\text { Aspectos culturales } \\
\text { (11 menciones) }\end{array}$ & $\begin{array}{l}\text { - Decepción amorosa } \\
\text { - Brujería } \\
\text { - Después de que le leyeron unas cartas y le pronosti- } \\
\text { caron mal augurio }\end{array}$ & $\begin{array}{l}\text { - Decepción amorosa } \\
\text { - Experiencias esotéricas (lectura de cartas, tarot) }\end{array}$ \\
\hline $\begin{array}{l}\text { Problemas laborales, } \\
\text { familiares, académicos } \\
\text { ( } 6 \text { menciones) }\end{array}$ & $\begin{array}{l}\text { - Problemas que se acumularon en su vida y que no } \\
\text { supo manejar }\end{array}$ & $\begin{array}{l}\text { - Pérdida de empleo } \\
\text { - Problemas familiares } \\
\text { - Tensiones en la escuela, con pareja y amigos }\end{array}$ \\
\hline Otros & $\begin{array}{l}\text { - Golpes en la cabeza } \\
\text { - "Impresión por asalto" } \\
\text { - Muerte de ser querido }\end{array}$ & $\begin{array}{l}\text { - Desmayos y golpes en la cabeza } \\
\text { - Comenzó a tener alucinaciones y delirios de persecución } \\
\text { - Complicaciones al nacer } \\
\text { - Muerte de ser querido }\end{array}$ \\
\hline
\end{tabular}


con enfermedades mentales; en tanto que el 88\% reportó haber vivido al menos una experiencia de rechazo social debido a su padecimiento:

"...la gente ya tiene la mentalidad, y no se le va a quitar esa imaginación de que uno es un loco y no nos va a bajar de locos y yo lo estoy padeciendo día a día, porque no puedo pedir justicia, no puedo gritar -yo tengo esto, porque menos me hacen caso, menos me hacen justicia- entonces así se le ponga veinte nombres bonitos, yo pienso que jamás van a cambiar su mente, que el loco no tiene derecho a nada". (Usuaria)

Las principales fuentes de estigma fueron la familia y el personal de salud, siendo las críticas, acusaciones, regaños, provocaciones, burlas, el uso de apodos y calificativos, las prácticas más comunes. Específicamente en el caso del personal de salud, algunas experiencias referidas tienen que ver con la falta de credibilidad y descalificación de la que son objeto, así como ciertas actitudes fatalistas con relación al pronóstico del padecimiento:

"Mi hermana me dice: -tú ya no puedes dialogar conmigo, tú ya no tienes opinión, tómate tu chocho y hazte a un lado". (Usuario)

"Por parte del personal de salud se dan estas actitudes hacia las personas con padecimientos mentales, se les desacreditan sus comentarios, se les trata con desinterés u hostilidad, se les devalúa mucho". (Psicóloga)

A continuación se incluye el relato de una informante quien, al participar en una intervención grupal con otras mujeres, recuerda una experiencia de abuso sexual, que en algún momento no denunció por la descalificación de la que fue objeto. En este sentido se observa cómo, al ser portadora de una enfermedad mental sumada a su condición de género, da lugar a ser una persona doblemente desacreditable:

"...cuando mi compañera contó que había sufrido abuso, entonces dije no, eso sí fue cierto, porque a mí me pasó, y ipor qué me lo callé?, porque si antes, cuando estaba chica, que digamos estaba bien de mis facultades no me creyeron, pues ahora menos. Por eso la verdad, no es justo, pero la trabajadora social dijo que no fue cierto, que es una invención de la chica por su enfermedad, pero no porque a mí me pasó y volví a sentirme otra vez sucia, cochina...". (Usuaria)

$\mathrm{Al}$ igual que ocurre en otros trastornos graves que involucran episodios psicóticos, en algunos casos el personal de salud suele tener ciertas dudas con respecto a los eventos narrados por los pacientes, sobre si son producto de un hecho real o consecuencia de dichos episodios o delirios, lo cual podría conducir a que estas personas sean objeto de una doble o triple estigmatización.

\section{Percepciones del personal de salud, acerca de las principales demandas de atención en salud mental y sectores más afectados de la población}

Los trastornos depresivos, la esquizofrenia, los trastornos de personalidad, la bipolaridad, la ansiedad, el abuso de sustancias y el alcohol fueron considerados por los entrevistados como las principales demandas de atención en su campo laboral. Asimismo mencionaron otros problemas asociados con ellos, tales como la violencia, el abuso sexual, la pobreza y el suicidio.

Al indagar sobre la percepción del personal de salud respecto a los sectores más afectados de la población por padecimientos psiquiátricos, el 39\% de los entrevistados consideró que cualquier persona puede ser proclive a tener un padecimiento de este tipo debido a que la causa de estos trastornos es genética, aunada a otras condiciones del medio ambiente como la inseguridad, la violencia y el estrés. El $34 \%$ de los entrevistados identificó a las personas de bajos recursos como el sector más afectado por las implicaciones que tiene el padecimiento (p.e. dificultades de acceso a la atención, vivir en zonas marginales y el elevado costo del medicamento y del tratamiento, etc.), particularmente personas en condiciones de abandono que viven en la calle y carecen de apoyo de todo tipo:

"...ese es el principal problema y como los medios de comunicación y esta situación política que tenemos actualmente nos llevan a que los jóvenes tengan menos interés en la vida, que se quieran suicidar... También las condiciones de pobreza en donde se da la prostitución, en donde se da la adicción, el alcoholismo, donde se dan las violaciones sexuales o el abuso sexual desde los padres ino?, entonces es muy triste la situación en este país y yo creo que todo el mundo, muy desagradable muy triste, no tienes para donde ir ino?... yo me siento muy este, muy limitada aquí con la gente con la que trabajo". (Trabajadora social)

Cabe señalar que el 7\% de los entrevistados hicieron referencia a las diferencias por género respecto a la prevalencia de trastornos psiquiátricos, según las cuales la depresión y la ansiedad son más frecuentes en las mujeres, en tanto que el consumo de alcohol y drogas predominó entre los hombres. Al respecto, un entrevistado consideró que en la práctica no existen las condiciones necesarias para brindar la atención desde una perspectiva de género:

"...desde mi opinión se hace investigación en esto de conocer el consumo, la problemática en la mujer consumidora, pero todavía no hay un modelo específico de tratamiento para ellas; entonces aquí tenemos un modelo pensado en población general y eso significa los hombres. Por ejemplo, llegan mujeres y no tenemos una sala que pueda contener a los niños, no existe eso, no hay paquetería para niños mientras la mamá está en tratamiento, no existe y también no es adecuado que la mamá entre con el niño al espacio de tratamiento, es pedirle que encargue a sus hijos con la comadre, con la hermana, o sea, generar cómo ella puede continuar con un proceso y que se le va a dificultar por los costos, ¿y que quién cuida al chamaco?, ¿quién va a irle a comprar el mandado?, ¿quién le haga la comida?, ¿quién recoja al niño inclusive, no? ese día que ella venga a tratamiento, es como ir resolviendo todas esas cosas de la vida cotidiana que le pueden imposibilitar su tratamiento". (Psicólogo)

Las consumidoras de alcohol y drogas suelen ser señaladas como responsables del padecimiento; algo similar ocurre con quienes son objeto de violencia en la pareja. Como señala un informante:

"sus quejas no se consideran confiables por una cuestión de género". (Psiquiatra)

\section{Dilemas y dificultades en la práctica laboral}

Las dificultades laborales son aquellas condiciones que el personal de salud percibe como un obstáculo para el desarrollo de su ejercicio profesional dentro del proceso de atención a la salud, por ejemplo, falta de equipo médico, de medicamentos, de recursos humanos, las cuales se relacionan con problemáticas institucionales que, en última instancia, tienen que ver con el estigma estructural, ya que la salud mental ocupa 
Estigma estructural, interseccionalidad, género y salud mental

una posición marginal respecto a la salud física en México. Asimismo, los dilemas implican la convergencia de dos soluciones posibles ante una determinada problemática, las que suelen ser incompatibles colocando al proveedor en una situación de conflicto que puede generar estrés o malestar. En el cuadro 5 se muestran algunos ejemplos con respecto a las dificultades percibidas, tales como las barreras institucionales y sus implicaciones para el desarrollo de las funciones del personal de salud. La falta de recursos humanos, materiales y financieros, ante la creciente demanda de servicios, sumado al escaso trabajo colaborativo entre el personal de salud y a la estructura vertical entre disciplinas constituyen las principales fuentes de malestar para los entrevistados:

"Las redes de comunicación entre los sectores de salud mental están totalmente deficientes, es más yo diría no existen....que si el hospital $X$ no quiso recibir que los reciba el hospital $Y$, y a veces ni siquiera con una hoja de referencia ... y así nos la vamos pasando. Entonces la comunidad no sabe qué hacer ante este tipo de situación". (Psiquiatra)

"...no hay como mucha comunicación, precisamente porque la gente se individualiza quiere hacer sus cosas muy independiente y no hay esa oportunidad... bueno algunas partes se salvan diría yo, pero desafortunadamente en este país, así nos han enseñado a trabajar ¿no?, cada quien ve por su vida". (Trabajadora social)

Para el abordaje de los dilemas, la estrategia fue indagar acerca de cuáles habían sido las experiencias más difíciles a las que habían hecho frente durante su quehacer profesional. Como se observa, las situaciones referidas tienen que ver con la intersección de distintas condiciones que generan un efecto de vulnerabilidades múltiples, por ejemplo: 1 . mujeres en situaciones específicas, embarazadas, menores de edad, sin redes de apoyo y viviendo en condiciones de violencia, 2. personas no heterosexuales o transgénero que solicitan atención y que para el proveedor pueden representar una trasgresión a sus propios valores e ideología, 3 . jóvenes adolescentes, con embarazo no deseado, sin red de apoyo, sin recursos económicos y con intento suicida, 4 . personas que presentan cierto deterioro cognitivo, sin recibir atención de ningún tipo, en condición de calle o recluidos en penales, 5 . personas de grupos étnicos o diferentes culturas que requieren condiciones específicas para su atención (p.e. intérprete lingüístico y cultural para la comprensión y manejo adecuado de su sintomatología), que además carecen de recursos económicos y de redes de apoyo, como se ilustra en el siguiente testimonio:

"...yo llegué a ese piso pasé a ver a mi compañera y en ese momento el paciente se le fue a golpes a una compañera sin mediar palabra. Ese paciente era de Somalia que no hablaba español, en el poco inglés que se le puede entender interpretamos. Ahí él nos narraba que en su mente había voces que le decían que la compañera quería que se muriera, que porque tenía SIDA, entonces su reacción de él fue desquitarse, pero yo te digo sin mediar palabra se le fue encima a golpes, afortunadamente yo llegaba y lo contuvimos, hablamos con él y se tranquilizó, y posteriormente ya llegó la guardia médica, se medicó y se le puso una sujeción gentil por dos horas...". (Enfermero)

Las personas con esquizofrenia que viven en la calle y permanecen en la indigencia son un sector olvidado por las instituciones. De igual manera ocurre con los consumidores de alcohol y drogas, quienes suelen ser objeto de maltrato e inatención en las instituciones porque de alguna manera se les responsabiliza por su padecimiento y se les atribuye socialmente la idea de promiscuidad y el llevar una vida desordenada. A estas personas no les queda más que iniciar el peregrinaje entre distintos lugares, siendo canalizados de institución en institución sin recibir atención en la mayoría de los casos. Estos ejemplos rebasan las competencias y habilidades del personal de la salud, algunas de ellas se derivan del estigma y la discriminación de carácter estructural, de manera que constituyen desafíos para la atención en salud mental.

\section{DISCUSIÓN}

La principal aportación de este trabajo, es que permitió dar cuenta de la naturaleza estructural del estigma y la discriminación hacia las enfermedades mentales, desde la perspectiva de las personas afectadas por estos padecimientos y del personal de salud que labora en distintos centros de atención en la Ciudad de México. ${ }^{5}$ En ese sentido, el enfoque basado en la interseccionalidad, ${ }^{12-14}$ fue de gran utilidad para el abordaje de la relación dinámica entre los sujetos sociales y ciertos determinantes sociales, lo cual permitió identificar las dificultades que suelen presentarse en el proceso de atención psiquiátrica.

El estigma y la discriminación hacia los padecimientos psiquiátricos fue la principal barrera que incide en la atención desde el punto de vista de los participantes y se presenta en distintos ámbitos a nivel clínico, familiar y social. $\mathrm{El}$ personal de salud fue reportado como la segunda fuente de estigma y discriminación, lo cual coincide con estudios previos. ${ }^{19-21} \mathrm{Al}$ respecto, $\mathrm{Szasz}^{22}$ refiere que existen algunos vacíos durante el proceso de formación médico-científica de las disciplinas y especialidades relacionadas con la salud, usualmente no se abordan los aspectos afectivos, así como los prejuicios y estereotipos negativos que se asocian con los padecimientos mentales, lo que en consecuencia da lugar a que estas prácticas se sigan reproduciendo durante la interacción médico-paciente.

Por otra parte, es importante considerar que por su actividad laboral, el personal de salud también es objeto de rechazo social, tanto de parte del gremio médico como de la sociedad en su conjunto, lo que en la bibliografía se conoce como el estigma por asociación,$^{20}$ lo que también tiene implicaciones en el proceso de atención.

Destaca el hecho de que los entrevistados, usuarios y personal de salud, hicieron referencia en sus testimonios a la presencia de múltiples condiciones de vulnerabilidad social, que si bien no se presentan en todos los casos, constituyen un obstáculo en la atención. Las condiciones de pobreza, la violencia, la falta de redes de apoyo y la comorbilidad con padecimientos físicos y mentales, fueron algunas de las más comunes y remiten a una serie de inequidades sociales de orden socioeconómico, político e histórico que forman parte del contexto biográfico del sujeto. 
Cuadro 5. Dificultades percibidas en su práctica laboral, según proveedores

\begin{tabular}{|c|c|c|}
\hline Dimensiones & Mujeres & Hombres \\
\hline $\begin{array}{l}\text { Barreras } \\
\text { institucionales } \\
\text { (57menciones) }\end{array}$ & $\begin{array}{l}\text { (41 menciones) } \\
\text { - Falta de infraestructura y espacios } \\
\text { - Lucha de intereses en el centro de atención } \\
\text { - Falta de delimitación de funciones del personal } \\
\text { - Falta de recursos humanos y materiales } \\
\text { - Falta de capacitación y supervisión del personal } \\
\text { - No se brindan verdaderas soluciones a la angustia o tensión familiar } \\
\text { - Desconocimiento sobre la salud mental en centros de atención pri- } \\
\text { - Naria } \\
\text { - } \text { - Falta de apalud trabajo colaborativo, prevalece la jerarquía en el equipo } \\
\text { - Tiempo insuficientico para las consultas } \\
\text { - Falta de colaboración interinstitucional }\end{array}$ & $\begin{array}{l}\text { (16 menciones) } \\
\text { - Falta de recursos humanos, materiales y financieros } \\
\text { - Ausencia de un modelo de tratamiento adecuado (tiempos de re- } \\
\text { cepción, frecuencia, burocracia) } \\
\text { - No hay atención integral } \\
\text { - Falta de capacitación del personal de salud en la atención } \\
\text { - Atraso en la atención oportuna como resultado trayectorias previas } \\
\text { - de búsqueda de atención en la población } \\
\text { - Falta de interés en la salud mental del personal }\end{array}$ \\
\hline $\begin{array}{l}\text { Usuario/paciente } \\
\text { (39 menciones) }\end{array}$ & $\begin{array}{l}\text { (23 menciones) } \\
\text { - Resistencia para recibir atención psiquiátrica } \\
\text { - Actitud sumisa ante el médico } \\
\text { - Falta de cumplimiento del reglamento institucional } \\
\text { - Falta de adherencia y abandono al tratamiento } \\
\text { - Nivel de deterioro cognitivo que dificulta el manejo del paciente } \\
\text { - Desconocimiento sobre la salud mental } \\
\text { - Barreras ocasionadas por la falta de recursos económicos } \\
\text { - Falta de apoyo familiar en los usuarios }\end{array}$ & $\begin{array}{l}\text { (16 menciones) } \\
\text { - Resistencia para recibir atención psiquiátrica } \\
\text { - Falta de adherencia y abandono al tratamiento } \\
\text { - Autoconcepto débil e inseguridad } \\
\text { - Violencia física y verbal } \\
\text { - El contacto con otros pacientes psiquiátricos les genera ansiedad } \\
\text { - Atención tardía } \\
\text { - Falta de información y cultura } \\
\text { - Barreras ocasionadas por la falta de recursos económicos }\end{array}$ \\
\hline $\begin{array}{l}\text { Familia } \\
\text { (33 menciones) }\end{array}$ & $\begin{array}{l}\text { - (23 menciones) } \\
\text { - Dalta de apoyo familiar } \\
\text { - Falta de cumplimiento del reglamento institucional } \\
\text { - Alta demanda de atención } \\
\text { - Incumplimiento de las indicaciones médicas } \\
\text { - Abandono de los usuarios } \\
\text { - Actitud hostil y de crítica hacia el usuario } \\
\text { - Carga emocional y estrés }\end{array}$ & $\begin{array}{l}\text { - Falta de apoyo familiar } \\
\text { - Desconocimiento del padecimiento del usuario } \\
\text { - Falta de información y cultura }\end{array}$ \\
\hline $\begin{array}{l}\text { Personal de salud } \\
\text { (27menciones) }\end{array}$ & $\begin{array}{l}\text { (21 menciones) } \\
\text { - Favoritismo hacia colegas } \\
\text { - Falta de preparación } \\
\text { - Falta de trabajo en equipo } \\
\text { - Experimentan mucha frustración en la atención } \\
\text { - Falta de compromiso e interés en su trabajo } \\
\text { - Tensiones y conflictos } \\
\text { - Falta de sensibilidad en la atención } \\
\text { - Desinterés en la capacitación } \\
\text { - Prácticas inadecuadas } \\
\text { - Desconocimiento sobre padecimientos psiquiátricos en la atención } \\
\text { - primaria } \\
\text { - Docha de egos } \\
\text { - Jerarquías, verticalidad en el modelo de atención }\end{array}$ & $\begin{array}{l}\text { - (6 menciones) } \\
\text { - Trabajo individualizado, no existe coordinación entre áreas y/o } \\
\text { - Falta de capacitación para el abordaje del paciente agitado } \\
\text { - Descalificación entre colegas } \\
\text { - Desgaste emocional y laboral } \\
\text { - Estigma del proveedor hacia los usuarios } \\
\text { - Incapacidad para abordar a familia y usuarios }\end{array}$ \\
\hline
\end{tabular}

Estigma y aspectos culturales (27 menciones)

Estado (21 menciones)

\section{(16 menciones)}

- Subestimación de la población general hacia su profesión

- Desconfianza de usuarios y familiares hacia el personal de salud

- Estigma y discriminación de algunos proveedores hacia los usuarios

- Descalificación entre las disciplinas de atención

- Ideas mágico-religiosas sobre la enfermedad mental en usuarios y familiares

- Falta de información en población general sobre enfermedades mentales y funciones del psiquiatra

(14 menciones)

- Falta de interés en la salud mental en las políticas públicas

- Inequidad social

- Precarias condiciones económicas de la población

- Recursos insuficientes para la atención psiquiátrica

- Ausencia de programas específicos para la atención de la población psiquiátrica (p.e. empleo, vivienda) en grupos vulnerados, principalmente en familias de bajos recursos económicos

- Carencia de apoyos sociales

Interacción (9 menciones)

\section{(4 menciones)}

- La medicación impide en ocasiones comunicarse con los usuarios - Manejo del paciente cuando está irritado o agitado (p.e agresiones físicas y verbales)

- Lidiar con el enojo de familiares y usuarios

- Se genera dependencia en la relación con usuario
(11 menciones)

- Prejuicios y mitos sobre la enfermedad mental en la sociedad

- Estigmatización de las personas con enfermedad mental

- Ideas mágico-religiosas sobre la enfermedad mental en usuarios y familiares

- Autoatención como primera búsqueda de ayuda (brujos, sacerdotes) genera un atraso en la atención médica

- Falta de información y de interés en salud mental por parte de la población

(7 menciones)

- Condiciones de pobreza en la población

- Los programas de salud se enfocan más en cifras que en dar solución a los problemas de salud

- Falta de empleo, principalmente para usuarios con adicciones

- Falta de modelos de tratamiento específicos para mujeres que incorporen en la práctica un enfoque de género

- La centralización de los servicios de atención psiquiátrica y el abandono de los más alejados

\section{(5 menciones)}

- Falta de disposición del personal de salud para escuchar a los pacientes

- Involucrase con la patología del paciente por falta de capacitación o contención

- Dificultad para establecer canales de comunicación adecuados con los usuarios y familiares 
Estigma estructural, interseccionalidad, género y salud mental

En ese sentido, la adopción del paradigma de interseccionalidad ${ }^{13,14}$ fue de gran utilidad ya que constituye una herramienta teórica para entender los procesos de estigmatización hacia los padecimientos psiquiátricos desde un enfoque más estructural que incluye el análisis de los determinantes sociales, entre ellos el género, lo que ofrece la posibilidad de trascender la construcción de conceptos aislados que refuerzan fronteras y oposiciones que contravienen la realidad social observada, a fin de generar estrategias dirigidas a la reducción de las condiciones desfavorables y de riesgo, ante las cuales ambos géneros están expuestos.

Desde esta perspectiva, el género remite a una serie de representaciones y prácticas sociales bajo las que se han definido y tratado las identidades de hombres y mujeres, en su aspecto relacional y en función de un contexto histórico y sociocultural particular, que en el caso de la salud influyen en la configuración de experiencias de enfermedad diferenciadas; en tanto que las políticas públicas tradicionalmente han empleado enfoques que, además de omitir la diversidad sociocultural, tampoco consideran las variaciones en la configuración y expresión de necesidades de salud entre la población.

Los usuarios y el personal de salud hicieron referencia en sus testimonios a las múltiples condiciones que obstaculizan la atención. Las condiciones de pobreza y la falta de recursos para la atención constituyen variables estructurales que inciden, al igual que el género, como variable transversal (p.e. experiencias de abuso sexual y violencia) en la manifestación de los padecimientos psiquiátricos dando cuenta de su complejidad.

El personal de salud suele experimentar impotencia e incertidumbre frente a los casos complejos en los que se presentan múltiples condiciones de vulnerabilidad social como el embarazo adolescente, el abuso de sustancias y las precarias condiciones de vida entre la población que atienden; lo que suele dar lugar a la desatención o a la indiferencia, sobre todo cuando el proveedor cuenta con varios años de desgaste laboral y emocional vinculado con su quehacer profesional.

Otro aspecto importante radica en que el $39 \%$ de los proveedores entrevistados consideró que todos los sectores de la población son susceptibles de tener un padecimiento de este tipo, aludiendo a los factores genéticos como la principal causa de la enfermedad. Al respecto, como señala Martínez-Hernáez, ${ }^{23}$ si bien en el discurso hay un reconocimiento en los proveedores de salud del enfoque multidimensional biopsicosocial, lo cierto es que en la práctica clínica continúa la tendencia a tratar la enfermedad mental desde una perspectiva exclusivamente biológica e individual, ignorando otros aspectos como la desigualdad social, las estructuras de poder y la cultura, así como la forma en que éstas afectan y determinan las condiciones de aparición, desarrollo y posibilidad de recuperación de la enfermedad.

Como se evidenció en los hallazgos, se observa un cierto desencuentro en la relación entre el personal de salud y los usuarios, mientras que existe un sector de éstos últimos que atribuyen ciertos aspectos mágico-religiosos al surgimiento del padecimiento psiquiátrico. El personal de salud considera que dichos aspectos culturales constituyen un obstáculo para lograr la adherencia terapéutica de los pacientes, por lo que suelen descalificar estas creencias, sin considerar que forman parte del universo simbólico y cultural de la población. Para autores como Kleinman ${ }^{24}$ estos saberes son de crucial importancia a fin de que la atención sea culturalmente adecuada tanto para los médicos como para los pacientes, de ahí la importancia de sensibilizar al personal de salud acerca de otorgar mayor importancia a los aspectos culturales, lo cual además de permitir entender la complejidad de los padecimientos psiquiátricos podría brindar una respuesta más acorde con las necesidades específicas de la población a la que brindan sus servicios.

Además de los aspectos culturales, el género juega un papel clave en el proceso de atención. ${ }^{25,26}$ En esta investigación, tan sólo el 7\% de los participantes del personal de salud hizo alusión a las diferencias por género, sin contar con el hecho de que en las prácticas concretas no suelen aplicarse los programas de género en la atención. En esa misma línea, estudios previos han reportado que el personal de salud no reconoce la violencia de género, ${ }^{27}$ en tanto que en esta investigación identificamos que uno de los riesgos posibles para el personal de salud es atribuir las experiencias de violencia o abuso sexual exclusivamente a la sintomatología psiquiátrica, sin considerar que podría tratarse de una demanda legítima por parte de los usuarios, en los casos que sufren un trastorno mental considerado grave, como es el caso de la esquizofrenia.

Es interesante observar que el personal de salud también se ve impactado por las inequidades de género, ejemplo de esto es el ingreso mensual reportado por los entrevistados, que sitúa a las mujeres por debajo del ingreso masculino, aun cuando el tiempo destinado a la atención es similar en ambos casos. Si bien este dato debe tomarse con precaución dado que existen otros aspectos del contexto específico de cada disciplina y de las actividades y funciones específicas del personal de salud, lo cierto es que encontramos estas diferencias, en las cuales, por cierto, hubo una mayor presencia de las mujeres en áreas relacionadas con el cuidado de la salud.

Finalmente, cabe señalar que si bien por medio de un estudio de corte cuantitativo es posible identificar una serie de variables que inciden en los padecimientos psiquiátricos, el aporte que brinda la perspectiva cualitativa radica en la posibilidad de dar cuenta no sólo de los aspectos que intervienen en el proceso de atención sino dar paso al análisis de las articulaciones y la complejidad de las interacciones entre diversas dimensiones o categorías sociales. Además de suscitar la reflexión no sólo respecto a la población de estudio, sino en otros sectores involucrados, por parte del personal de salud e incluso de quienes se interesan en el estudio de estas áreas, valorando los aspectos ideológicos y culturales que subyacen a las prácticas sociales. 
Cabe señalar que este trabajo formó parte de un estudio más amplio, de manera que no fue diseñado específicamente para abordar el género y la salud mental desde una perspectiva interseccional. Sin embargo, durante el proceso de análisis de la información obtenida en la población de estudio y en la búsqueda de referentes teóricos para la interpretación de los hallazgos, encontramos que este enfoque es de gran utilidad ya que enfatiza la importancia de la interrelación entre los determinantes sociales y que el género constituye una variable transversal fundamental alrededor del proceso de salud-enfermedad-atención. Por lo tanto, consideramos que esta propuesta podría enriquecer la investigación en esta área, principalmente en contextos latinoamericanos, donde existen marcadas desigualdades sociales que no pueden quedar al margen del análisis de las condiciones de salud de la población.

\section{AGRADECIMIENTOS}

Las autoras expresamos nuestro reconocimiento a los informantes que compartieron su experiencia y sus puntos de vista durante el estudio, por su disposición e interés para mejorar la calidad de la atención en el campo de la salud mental. Asimismo, expresamos nuestra gratitud al doctor Duncan Pedersen, del Institut Universitaire en Santé Mentale Douglas, Montreal, Quebec, por impulsar la iniciativa de investigación sobre Estigma y Enfermedad mental en México, sin la cual no habría sido posible esta colaboración internacional. A los Fondos Sectoriales en Salud por apoyar financieramente este proyecto (69261), a las instituciones participantes y al equipo de entrevistadores por su compromiso e interés durante el proceso de recolección de la información, así como a Xanat Aguilera (servicio social).

\section{REFERENCIAS}

1. Stuart H, Arboleda-Florez J, Sartorius N. Paradigms lost. Fighting stigma and the lessons learned. Oxford: Oxford University Press; 2012.

2. Bos A, Pryor J, Reeder G, Stutterheim S. Stigma: advances in theory and research. Basic Applied Social Psychology 2013;35(1):1-9.

3. Goffman E. Stigma: Notes on the management of spoiled identity. Englewood Cliffs: Prentice Hall; 1963.

4. Corrigan P. Illness self-management strategies. A guideline developed for the behavioural health recovery management project. Illinois: University of Chicago Center for Psychiatric Rehabilitation; 2008.

5. Link B, Phelan P. Conceptualizing Stigma. Annual Review Sociology 2001;27:363-385.

6. Corrigan P, Markowitz F, Watson A. Structural Levels of Mental Illness. Stigma and Discrimination. Schizophrenia Bulletin 2004;30:481-491.

7. Herek G. Sexual stigma and sexual prejudice in the United States: A conceptual framework. En: Hope D (ed.). Contemporary perspectives on lesbian, gay and bisexual identities: The 54th Nebraska Symposium on Motivation. Nueva York: Springer; 2009.

8. Flores F, Mora-Rios J. Pobres, enfermas y locas, una historia de vulnerabilidades acumuladas. En: Montero E (ed.). Ecología social de la pobreza: Impactos psicosociales, desafíos multidisciplinarios. México: UNAM; 2010.
9. Romero M, Saldívar G, Loyola L, Rodríguez E et al. Inequidades de género, abuso de sustancias y barreras al tratamiento en mujeres en prisión. Salud Mental 2010;33(6):499-506.

10. Miller K, Rasmussen A. War exposure, daily stressors, and mental health in conflict and post-conflict settings: Brindging the divide between trauma-focused and psychosocial frameworks. Social Science Medicine 2010;70:7-16.

11. Espinosa LM, Mora-Rios J, Valenzuela M. Saberes y trayectorias de atención a la salud de poblaciones vulneradas en México: abordaje interdisciplinario. Revista Saúde Sociedade 2013;22(25):590-602.

12. Crenshaw K: Mapping the Margins: Intersectionality, identity politics and violence against women of colour. Critical Race Theory: The Key Writings that Informed the Movement. Nueva York: New York Press; 1995.

13. Hankivsky O, Reid C, Cornier R, Varcoe C et al. Exploring the promises of intersectionality for advancing women's health research. International J Equity Health 2010;9(5):1-15.

14. Hankivsky O. Women's health, men's health, and gender and health: Implications of intersectionality. Social Science Medicine 2012;74:1712-1720.

15. Pedersen D. Estigma y exclusión social en la enfermedad mental: Apuntes para el análisis y diseños de intervenciones. Acta Psiquiátrica Psicológica América Latina 2009;55(1):39-50.

16. Mora-Ríos J, Natera G, Bautista-Aguilar N, Ortega-Ortega M. Estigma público y enfermedad mental. Una aproximación desde la teoría de las representaciones sociales. En: Flores F (Coord.). Representaciones sociales y contextos de investigación con perspectiva de género. México: CRIM-UNAM; 2013.

17. Mora-Ríos J, Ortega-Ortega M, Natera G. Autoestigma en usuarios de servicios psiquiátricos y su relación con variables sociodemográficas, clínicas y psicosociales. Acta Psiquiátrica Psicológica América Latina 2013;59(3):147-158.

18. Atlas-ti 7.0 for Windows [computer software]. (2012). Berlin: Cincom Systems, Inc. (5 de febrero de 2014).

19. Lauber C, Nordt C, Braunschweig C, Rossler W. Do mental health professionals stigmatize their patients? Acta Psychiatr Scand 2006;(Suppl):429:51-59.

20. Schulze B. Stigma and mental health professionals: A review of the evidence on an intricate relationship. International Review Psychiatry 2007;19:137-155.

21. Wahl O, Aroesty-Cohen E. Attitudes of mental health professionals about mental illness a review of the recent literature. J Community Psychology 2009;38:49-62.

22. Szasz, Ivonne. "Género y salud. Propuesta para el análisis de una relación compleja". En: Bronfman M, Castro R (cords.). Salud, cambio social y política. Perspectivas desde América Latina. México: Instituto Nacional de Salud Pública y Foro Internacional de Ciencias Sociales y Salud; 1999; pp.109-121.

23. Martínez-Hernáez A. Más allá de la rehabilitación psicosocial. Metáforas de exclusión y tareas de inclusión. Cad Bras Saúde Mental 2009;1:1-12.

24. Kleinman A. Anthropology and psychiatry. The role of culture in a cross-cultural research on illness. British J Psychiatry 1987;151:447-454.

25. Wang J, Fick G, Adair C, Lai D. Gender specific correlates of stigma toward depression in a Canadian general population simple. J Affective Disorders 2007;103:91-97.

26. Carpenter-Song E, Chu E, Drake R, Ritsema M et al. Ethno-cultural variations in the experience and meaning of mental illness and treatment: implications for access and utilization. Transcultural Psychiatry 2010;47(2):224-251.

27. Coll-Vinent B, Echeverría T, Farras U, Rodríguez D et al. El personal sanitario no percibe la violencia doméstica como un problema de salud. GacSanit 2008;22(1):7-10.

Artículo sin conflicto de intereses 